\title{
Review Article \\ Tregitope Peptides: The Active Pharmaceutical Ingredient of IVIG?
}

\author{
Anne S. De Groot, ${ }^{1,2}$ Leslie Cousens, ${ }^{2}$ Federico Mingozzi, ${ }^{3,4}$ and William Martin ${ }^{2}$ \\ ${ }^{1}$ Institute for Immunology and Informatics, University of Rhode Island, Providence, RI, USA \\ ${ }^{2}$ EpiVax, Inc., 146 Clifford Street, Providence, RI, USA \\ ${ }^{3}$ Genethon, Evry, France \\ ${ }^{4}$ University Pierre and Marie Curie, Paris, France
}

Correspondence should be addressed to Anne S. De Groot; annied@epivax.com

Received 12 July 2013; Accepted 17 October 2013

Academic Editor: Darren R. Flower

Copyright (C) 2013 Anne S. De Groot et al. This is an open access article distributed under the Creative Commons Attribution License, which permits unrestricted use, distribution, and reproduction in any medium, provided the original work is properly cited.

Five years ago, we reported the identification and characterization of several regulatory T-cell epitopes (now called Tregitopes) that were discovered in the heavy and light chains of IgG (De Groot et al. Blood, 2008). When added ex vivo to human PBMCs, these Tregitopes activated regulatory T cells (Tregs), increased expression of the transcription factor FoxP3, and induced IL10 expression in $\mathrm{CD}^{+}{ }^{+} \mathrm{T}$ cells. We have now shown that coadministration of the Tregitopes in vivo, in a number of different murine models of autoimmune disease, can suppress immune responses to antigen in an antigen-specific manner, and that this response is mediated by Tregs. In addition we have shown that, although these are generally promiscuous epitopes, the activity of individual Tregitope peptides is restricted by HLA. In this brief report, we provide an overview of the effects of Tregitopes in vivo, discuss potential applications, and suggest that Tregitopes may represent one of the "active pharmaceutical ingredients" of IVIg. Tregitope applications may include any of the autoimmune diseases that are currently treated almost exclusively with intravenous immunoglobulin G (IVIG), such as Chronic Inflammatory Demyelinating Polyneuropathy (CIDP) and Multifocal Motor Neuropathy (MMN), as well as gene therapy and allergy where Tregitopes may provide a means of inducing antigen-specific tolerance.

\section{Introduction}

In recent work [1], we identified an important trigger for the expansion and activation of regulatory $\mathrm{T}$ cells (Tregs), which are $\mathrm{T}$ cell epitopes contained in the framework sequences of immunoglobulin G (IgG). Further studies suggested that these peptides were natural $T$ regulatory cell epitopes (Tregitopes) that may explain, at least in part, the tolerance-inducing effects of polyclonal immunoglobulin when delivered as a therapy (intravenous immunoglobulin or IVIG). The defining characteristics of Tregitopes were that they (i) stimulated $\mathrm{CD} 4^{+}, \mathrm{CD} 25^{\text {hi }}$, and FoxP3 ${ }^{+}$T cells; (ii) suppressed effector T-cell responses to other antigens in suppressor assays; and (iii) were associated with $\mathrm{T}$ cell IL-10 production in vivo and in vitro [1]. Subsequently, Tregitope peptides have been shown to replicate the effects of IVIG in mouse models of
Multiple Sclerosis (EAE), allergy, and asthma, confirming our primary observations [2-4]. Consistent with their intrinsic immunosuppressive property, Tregitope peptides administered in complete or incomplete Freund's Adjuvant (CFA or IFA) suppress immune responses to coadministered antigens, but are not immunogenic per se [5].

While coadministration of Tregitope peptides with target antigen(s) effectively suppresses antigen-specific immune responses [6], Tregitope peptides are also particularly active in animal models on their own, if they are given during the acute phase of inflammation. For example, upon onset of diabetes Tregitope peptides formulated in IFA and delivered as a single dose to NOD mice (intraperitoneally) effectively suppressed diabetes in 58 percent of the mice for 25 weeks [6]. In a rigorous and independent NOD study conducted by the National Institute of Diabetes and Digestive and 
TABLE 1: Partial list of previously identified human IgG tregitopes.

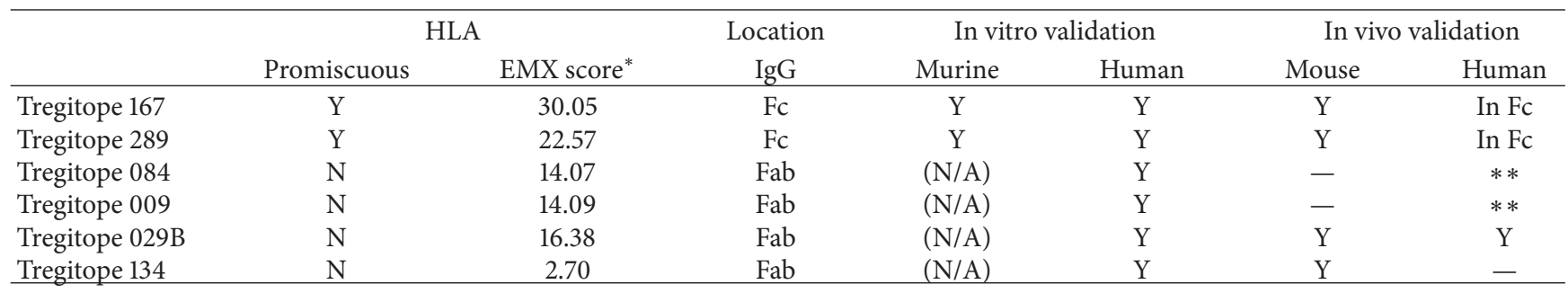

* EMX Score: EpiMatrix-predicted MHC binding promiscuity; correlates with T-cell response.

N/A: no murine homolog.

** Other data, not able to disclose.

In Fc: fc Fusion proteins have both Tregitope 167 and 289 present. See D. W. Scott publications.

Kidney Diseases (NIDDK), Tregitope peptides were the only novel therapy of six tested that exhibited "notable trends;" three diabetic mice remitted entirely, all of which were in the Tregitope peptide treatment groups. None of the other therapies tested (such as DT22669 (DiaKine), Aralast NP (Baxter Healthcare), ISO-092 (Feinstein Institute for Medical Research), Celastrol (Pi and Pi Technology), and PGCGLP-1 (PharmaINCorporation)) resulted in the prolonged remission seen with Tregitope peptides in this aggressive model of diabetes [7]. In addition, Tregitope peptides delivered in adeno-associated virus (AAV) ten days prior to TNBS (2,4,6 trinitrobenzenesulfonic acid-induced colitis) treatment were sufficient to suppress inflammatory bowel disease (and induce immigration of Tregs to the intestine) in this model of autoimmune disease [8].

\section{Tregitopes: What Are They?}

2.1. Natural and Induced Regulatory T Cells and Tolerance. It has become increasingly clear that $\mathrm{CD} 4{ }^{+} \mathrm{CD} 25^{+} \mathrm{FoxP}^{+}$ Tregs are an important component of immune regulation [9]. Autoreactive $\mathrm{T}$ cells with moderate $\mathrm{T}$-cell receptor affinity may escape deletion in the thymus to circulate where they function as "natural" regulatory T cells (nTregs) [10]. Two distinct Treg subsets are described in the literature: natural nTregs specific for self-epitopes and generated by highavidity selection in the thymus, and inducible iTregs that are derived from conventional $\left(\mathrm{CD}^{+}\right.$, FoxP3 $\left.{ }^{-}\right) \mathrm{T}$ cells following stimulation in the periphery $[11,12]$. nTregs can induce the conversion of conventional $\mathrm{T}$ cells to iTregs via cytokinedependent and -independent mechanisms, a process called infectious tolerance $[13,14]$.

It has been surmised that autologous proteins contain nTregitopes; however, few of these have been mapped. Immunoglobulin $G$ has been known to exhibit tolerogenic properties for decades, and a number of previous publications have alluded to the potential presence of regulatory or "suppressor" epitopes in IgG constant domains, whether located to the constant $(\mathrm{Fc})$ or binding region (Fab). For example, Baxevanis et al. described a tolerizing effect of Ig Fc that was localized to the $\mathrm{CH} 2$ region, consistent with the location of several Tregitopes [15]; a peptide isolated from the Fab region of an anti-idiotypic peptide (which overlaps one of the subsequently identified Tregitopes) suppressed Systemic Lupus
Erythematosis in humans and in mice [16, 17]; a peptide derived from the highly conserved J region framework was shown by Warnke et al. to induce Tregs to expand and suppress immune responses in suppressor assays [18].

We perform immunogenicity studies for a range of clients in the preclinical phase of monoclonal antibody development $[19,20]$. Using immunoinformatics tools (EpiMatrix [21]), now comprised in the ISPRI toolkit [20], we scanned the constant domains of human IgG and found that these and other regions of immunoglobulin $\mathrm{G}$ contained putative epitopes that were predicted to bind to more than one HLA and that were highly conserved in existing databases of IgG sequences. We hypothesized that they would serve to induce Tregs rather than $\mathrm{T}$ effector $\mathrm{T}$ cells, and that this might explain why some monoclonal antibodies that contain "foreign" (not seen in thymic development) sequences might not generate immune responses. Whether these Tregitope peptides induce nTregs or iTregs (peripheral, inducible Tregs), or both, remains to be determined. Nonetheless, we note that monoclonal antibodies that contain the full complement of Tregitopes are less likely to trigger immune responses despite hypervariable sequences that are comprised of de novo sequences and thus might appear "foreign" [22].

2.2. Tregitopes Are Found in IgG and in Fab as well as in Fc. As shown in Table 1, Tregitopes are promiscuous epitopes (as predicted by EpiMatrix) and are located in the Fc and the Fab regions of IgG. In contrast, Tregitopes are not found in other antibody isotypes (IgE, $\operatorname{Ig} A$, and $\operatorname{IgM}$ ). It is interesting to note that IgG but not IgM was found to induce tolerance in seminal studies of tolerance to haptens, performed by Golan and Borel [23]. Tolerance induction to IgG Fc-conjugated antigens has been extensively described by Scott et al. [24-26], among others [27], who very clearly demonstrated that HLA class II was involved [28] and that the effect did not require Fc receptor binding [29], which supports our hypothesis that tolerance is induced by the presence of HLA class II-restricted Tregitopes. As described above, Tregitopes are also found in IgG Fab, which can explain why Fab is as effective as IgG in inducing Tregs [30]. The Tregitope hypothesis is distinct from the work of Anthony et al. [31], who suggest IVIG-induced tolerance is mediated through sialylated $\mathrm{Fc}$ that initiate an anti-inflammatory signaling cascade through the lectin receptor SIGN-R1 or DC-SIGN. Tregitopes may, however, 


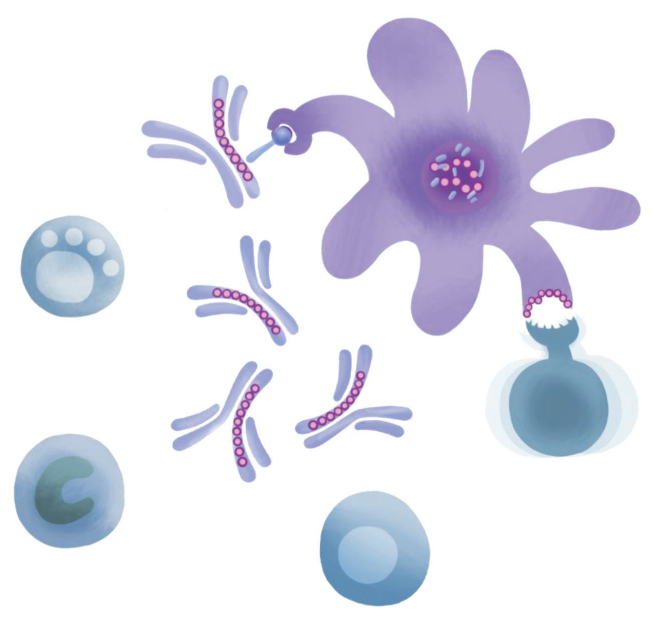

Figure 1: Potential IgG (and Tregitope) Mechanisms of Action. From left to right, IVIG has been demonstrated to affect the cells of the innate and adaptive immune system including NK cells, macrophages, $\mathrm{B}$ cells, $\mathrm{T}$ cells, dendritic cells and other antigen presenting cells.

explain the importance of binding to DC-SIGN, since that surface molecule traffics bound antigens directly to the class II processing and presentation pathway in dendritic cells.

2.3. Are Tregitopes the "Active Pharmaceutical Ingredient (API)" of IVIG? The effects of IVIG have been attributed to a wide array of mechanisms (Figure 1). Additional mechanisms include the formation of immune complexes [32]; interaction of sialylated $\mathrm{Fc}$ with a novel macrophage receptor DC-SIGN [33]; blockade of Fc receptors leading to clearance of antiself antibodies [34]; immunomodulation via anti-idiotypic interactions [35]; inhibition of complement-mediated tissue damage [36]; direct modulation of cytokine expression by leukocytes and endothelial cells; inhibition of superantigenmediated T-cell activation [31, 37, 38]; and induction of nTregs [11]. IVIG has recently been shown to be associated with modulation of the regulatory T-cell axis, reduction of IL17 [39], and enhancement of the suppressive function of Tregs [40]. In more recent studies by Massoud et al., the induction of Tregs by IVIG was shown to be dependent on IgG binding to a surface receptor called DCIR, followed by internalization and processing [41]. Many of these observed effects are consistent with the proposed Tregitope mechanism of action, as Tregitopes are peptides that would result from IgG internalization and processing, and have been associated with expansion of existing Tregs and induction of iTregs. Internalization of IgG (from IVIG), presentation of Tregitopes in the context of MHC class II, and expansion of Tregitope-specific Tregs would be consistent with recent observations that administration of IVIG induces expansion of Tregs and IL10 secretion in vivo, in animals and in humans $[9,11,42,43]$.

\section{Overview of Recent Tregitope Studies}

3.1. Proposed Mechanism of Action. Since our original description of Tregitopes in 2008, we have significantly advanced our understanding of the Tregitope mechanism of action, leading to our working hypotheses: (1) Tregitope effects are contingent upon major histocompatibility complex (MHC) class II-mediated presentation to a $\mathrm{T}$ cell by an antigen-presenting cell (APC), (2) Tregs recognize Tregitopes presented in the context of MHC II and are activated, (3) these activated Tregs produce IL-10 and interact with the APC to reinforce the development of a tolerogenic phenotype, and (4) these tolerogenic APCs and/or Tregs act on adjacent antigenspecific effector $\mathrm{T}$ cells to suppress their effector responses and induce antigen-specific Tregs. Published studies on Tregitopes by our own group and our collaborators corroborate the proposed mechanism, including in vivo studies, a few of which are summarized in Section 4.

3.2. Comparison of Tregitopes to IVIG in EAE. In separate in vivo and in vitro studies reviewed previously [4], we collaborated with the laboratory of Khoury and Elyaman to compare Tregitope peptide treatment to IVIG in the EAE model of Multiple Sclerosis.The in vivo study evaluates the capacity of IgG-derived Tregitope peptides to generate antigen-specific adaptive tolerance induction to MOG35-55 epitopes. In that study, mice were presensitized by MOG immunization. EAE disease was established and treated with IVIG or human IgG Tregitope peptides 167 and 289 in saline [4]. A tolerogenic effect of Tregitope peptides on immune responses to the MOG35-55 epitopes was observed in vitro and in vivo. These results are consistent with previous reports by Legge et al. [44] who showed induction of Tregs by IgG fusion proteins with multiple sclerosis antigens.

3.3. Tregitope Peptides Suppress $C D 4^{+}$T-Cell Responses and Are Not Immunogenic per se. In a recent paper, we demonstrated that Tregitope peptides are not immunogenic in vivo even when emulsified with potent adjuvants, such as IFA or CFA [5]. Moreover, in vivo administration of Tregitope peptides with IFA or CFA does not induce Th1 or Th2 cytokine expression under restimulation conditions in vitro. We investigated tolerance induction by codelivering Tregitope peptides with OVA using B cells. When B cells were pulsed with OVA and Tregitope peptides and transferred into naïve mice, we found that cellular and humoral immune responses to the OVA were suppressed as a result of their ability to induce Tregs and the absence of immunogenicity in the context of strong adjuvants [5].

3.4. Tregitope-Induced Tregs Also Modulate $C D 8^{+}$T-Cell Responses. Immune responses directed against viral capsid proteins constitute a main safety concern in the use of AAV as a gene transfer vector in humans. Using Tregitope peptides, we showed that it is possible to modulate $\mathrm{CD} 8^{+}$ T-cell responses to several viral antigens in vitro. Incubation of peripheral blood mononuclear cells with Tregitope peptides and viral epitope peptides triggered proliferation of $\mathrm{CD}^{+} \mathrm{CD} 25^{+} \mathrm{FoxP}^{+} \mathrm{T}$ cells that suppressed killing of 
target cells loaded with MHC class I antigens in an antigenspecific fashion, through a mechanism that required cell-tocell contact. Expression of a construct encoding for the AAV capsid structural protein fused to Tregitope peptides resulted in reduction of $\mathrm{CD}^{+} \mathrm{T}$-cell reactivity against the AAV capsid following immunization with a capsid-expressing adenoviral vector. This was accompanied by increased frequency of $\mathrm{CD} 4^{+} \mathrm{CD} 25^{+} \mathrm{FoxP}^{+} \mathrm{T}$ cells in spleens and decreased inflammatory infiltrates in injected tissues. This study demonstrated the feasibility of modulating $\mathrm{CD}^{+}$T-cell reactivity to an antigen using Tregitopes.

\section{Conclusion}

4.1. Can Tregitope Peptides Replace IVIG? IVIG is a pooled blood product from 10,000 or more donors. Many adverse effects (AEs) associated with IVIG administration are mild and transient, including headache, flushing, malaise, fever, chills, fatigue, nausea, vomiting, diarrhea, blood pressure changes, tachycardia, and anaphylactic reactions. Rare but more severe AEs include acute renal failure, thromboembolic events, skin-related effects including toxic epidermal necrolysis, and aseptic meningitis [45]. Many of these effects have been attributed to the targeting function of individual antibodies in the polyclonal immunoglobulin mixture. In general, however, IVIG is considered relatively safe. Thus, the main incentives to seek alternatives are either economic (to reduce the cost to patients and insurers) or to preserve IVIG supply for patients with primary immunodeficiencies who require the functional antibody component of IVIG to protect against infections. Tregitopes may, in part, explain the mechanism by which IVIG exerts its tolerogenic effect. Where those effects are Treg-mediated, Tregitopes might serve as an alternative IVIG for autoimmune conditions that may be safer (due to the absence of the functioning antibody component) and more effective.

If the induction of Tregs by IVIG can be attributed to Tregitopes, then a number of autoimmune diseases for which IVIG therapy is currently used, on-label and off, may be appropriate targets for immunomodulatory formulations that only contain Tregitope peptides. Two examples of disease in which IVIG is the predominant immunotherapy include Chronic Inflammatory Demyelinating Polyneuropathy (CIDP) and Multifocal Motor Neuropathy (MMN). Introduction of Tregitope peptides as alternatives to IVIG would also have a dramatic impact on the demand for IVIG for immune modulation therapy [11, 43, 45]. IVIG also acts rapidly and effectively in Immune Thrombocytopenic Purpura (ITP), Kawasaki Syndrome (KS), polymyositis, dermatomyositis, neurological syndromes such as GuillainBarré and CIDP, cases of severe steroid-dependent asthma, and many others $[46,47]$. Tregitope-peptide therapy may be an attractive alternative to the systemic immune suppression treatments that are sometimes used for these conditions.

4.2. Additional Tregitope Applications. Replacement of a pooled-donor, blood-derived product for which the mechanism of action is not certain with a therapeutic product for which the Active Pharmaceutical Ingredient (API) is

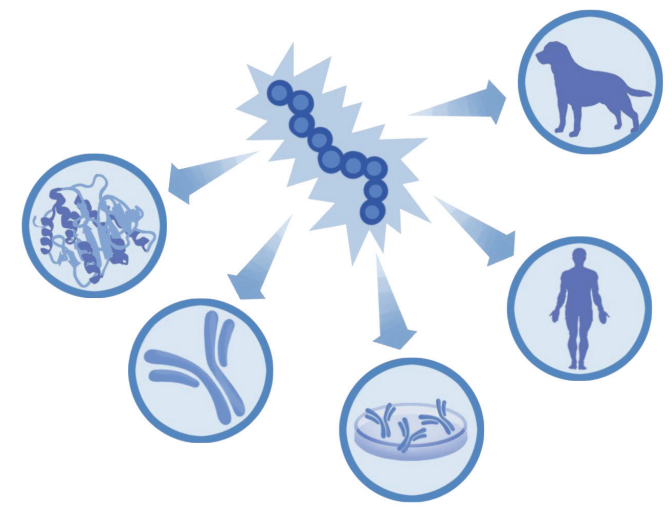

FIgURE 2: The broader relevance of Tregitopes is shown from left to right (counter clockwise). Tregitopes may be incorporated into protein drugs and monoclonal antibodies, to suppress antitherapeutic protein immune responses. Removing Tregitopes from autologous antigens would improve the ability of protein engineers to develop effective antibodies for target (autologous) antigens, and engineering Tregitope-depleted antibodies may improve the delivery of vaccine antigens. As described in this paper, Tregitopes may represent a new therapeutic option for autoimmune disease. In addition, Tregitopes could be used to suppress autoimmune diseases in companion animals.

precisely defined would represent a major step forward for the field of autoimmune disease therapy. In addition, since Tregitopes appear to be able to induce antigen-specific tolerance (induced Tregs that are specific to coadministered proteins), the possibility of tailoring the Tregitope therapy to specific autoimmune diseases would represent an additional advantage for clinical applications (Figure 2). One could imagine allergen-specific treatments using combinations of Tregitope peptides and allergen proteins or peptides, and treatment for autoimmune diseases such as diabetes, which would rely on coadministration of Tregitope peptides and diabetes antigens. The idea of specifically generating MOGreactive Tregs is also particularly attractive for the therapy of Multiple Sclerosis where, based on our data in the EAE mouse model, we believe that Tregitope peptides induce antigenspecific adaptive tolerance. Tregitopes may also have even broader applications in protein therapeutics, animal health, and blood factor or enzyme replacement therapy.

Treatment of many autoimmune diseases relies on immunosuppressive therapy rather than on treatments directed toward restoring a balance between effector and regulatory immune responses. Given that Tregitope peptides appear to induce adaptive tolerance in a mouse model, the next consideration is to evaluate the optimal formulation and determine the best time course of the tolerance induction, in addition to measuring the duration of response, the dose required, the safety and toxicity of the treatment (vis-à-vis other immune responses), and optimal formulation/route of administration. Our preliminary studies suggest that adaptive tolerance induction may be within reach, raising hopes that we are on the right path for the development of an effective immunotherapy-based approach to autoimmune disease. 


\section{Glossary}

Tregs: $\quad$ Regulatory T cells

Tregitopes: Treg epitopes (first described for IgG in 2008)

nTregs: Natural T regulatory cells

iTregs: Inducible T regulatory cells.

\section{Acknowledgments}

The authors gratefully acknowledge the thoughtful discussions and expert insights of Dr. David Scott (Department of Medicine, Uniformed Services University of Health Sciences) and Dr. Bruce Mazer (McGill University Health Center, Meakins Christie Laboratories), and the assistance of Kelsey Confreda (manuscript assistant) and Genevieve De Groot (illustrations).

\section{References}

[1] A. S. De Groot, L. Moise, J. A. McMurry et al., "Activation of natural regulatory T cells by IgG Fc-derived peptide 'Tregitopes," Blood, vol. 112, no. 8, pp. 3303-3311, 2008.

[2] L. P. Cousens, N. Najafian, F. Mingozzi et al., "In vitro and in vivo studies of IgG-derived Treg epitopes (Tregitopes): a promising new tool for tolerance induction and treatment of autoimmunity," Journal of Clinical Immunology, vol. 33, no. 1, pp. 43-49, 2013.

[3] L. P. Cousens, R. Tassone, B. D. Mazer, V. Ramachandiran, D. W. Scott, and A. S. De Groot, "Tregitope update: mechanism of action parallels IVIg," Autoimmunity Reviews, vol. 12, no. 3, pp. 436-443, 2012.

[4] W. Elyaman, S. J. Khoury, D. W. Scott, and A. S. De Groot, "Potential application of Tregitopes as immuno-modulating agents in multiple sclerosis," Neurology Research International, vol. 2011, Article ID 256460, 6 pages, 2011.

[5] Y. Su, R. Rossi, A. S. De Groot, and D. W. Scott, "Regulatory T cell epitopes (Tregitopes) in IgG induce tolerance in vivo and lack immunogenicity per se," Journal of Leukocyte Biology, vol. 94, no. 2, pp. 377-383, 2013.

[6] L. P. Cousens, Y. Su, E. McClaine et al., "Application of IgGderived natural treg epitopes (IgG Tregitopes) to antigenspecific tolerance induction in a murine model of type 1 diabetes," Journal of Diabetes Research, vol. 2013, Article ID 621693, 17 pages, 2013.

[7] C. W. Grant, C. M. Moran-Paul, S. K. Duclos, D. L. Guberski, G. Arreaza-Rubín et al., "Testing agents for prevention or reversal of type 1 diabetes in rodents," PLoS ONE, vol. 8, no. 8, Article ID e72989, 2013.

[8] S. van der Marel, A. Majowicz, K. Kwikkers et al., "Adenoassociated virus mediated delivery of Tregitope 167 ameliorates experimental colitis," World Journal of Gastroenterology, vol. 18, no. 32, pp. 4288-4299, 2012.

[9] A. Ephrem, S. Chamat, C. Miquel et al., "Expansion of $\mathrm{CD} 4{ }^{+} \mathrm{CD} 25^{+}$regulatory $\mathrm{T}$ cells by intravenous immunoglobulin: a critical factor in controlling experimental autoimmune encephalomyelitis," Blood, vol. 111, no. 2, pp. 715-722, 2008.

[10] J. A. Bluestone and A. K. Abbas, "Natural versus adaptive regulatory T cells," Nature Reviews Immunology, vol. 3, no. 3, pp. 253-257, 2003.
[11] M. S. Maddur, S. Othy, P. Hegde et al., "Immunomodulation by intravenous immunoglobulin: role of regulatory T cells," Journal of Clinical Immunology, vol. 30, no. 1, pp. S4-S8, 2010.

[12] M. Miyara and S. Sakaguchi, "Human FoxP3 ${ }^{+} \mathrm{CD}^{+}$regulatory T cells: their knowns and unknowns," Immunology and Cell Biology, vol. 89, no. 3, pp. 346-351, 2011.

[13] H. Jonuleit, E. Schmitt, H. Kakirman, M. Stassen, J. Knop, and A. H. Enk, "Infectious tolerance: human $\mathrm{CD} 25^{+}$regulatory $\mathrm{T}$ cells convey suppressor activity to conventional CD $4^{+} \mathrm{T}$ helper cells," Journal of Experimental Medicine, vol. 196, no. 2, pp. 255260, 2002.

[14] D. M. Gravano and D. A. A. Vignali, "The battle against immunopathology: infectious tolerance mediated by regulatory T cells," Cellular and Molecular Life Sciences, vol. 69, no. 12, pp. 1997-2008, 2012.

[15] C. N. Baxevanis, C.-D. G. Ioannides, G. J. Reclos, and M. Papamichail, "Evidence for distinct epitopes on human IgG with T cell proliferative and suppressor function," European Journal of Immunology, vol. 16, no. 8, pp. 1013-1016, 1986.

[16] Z. M. Sthoeger, A. Sharabi, Y. Molad et al., "Treatment of lupus patients with a tolerogenic peptide, hCDR1 (Edratide): immunomodulation of gene expression," Journal of Autoimmunity, vol. 33, no. 1, pp. 77-82, 2009.

[17] Z. Sthoeger, H. Zinger, A. Sharabi, I. Asher, and E. Mozes, "The tolerogenic peptide, hCDR1, down-regulates the expression of interferon- $\alpha$ in murine and human systemic lupus erythematosus," PLoS ONE, vol. 8, no. 3, Article ID e60394, 2013.

[18] M. Warncke, M. Buchner, G. Thaller et al., "Control of the specificity of T cell-mediated anti-idiotype immunity by natural regulatory T cells," Cancer Immunology, Immunotherapy, vol. 60, no. 1, pp. 49-60, 2011.

[19] V. Jawa, L. P. Cousens, M. Awwad, E. Wakshull, H. Kropshofer, and A. S. De Groot, "T-cell dependent immunogenicity of protein therapeutics: preclinical assessment and mitigation," Clinical Immunology, vol. 149, no. 3, pp. 534-555, 2013.

[20] A. S. De Groot and W. Martin, "Reducing risk, improving outcomes: bioengineering less immunogenic protein therapeutics," Clinical Immunology, vol. 131, no. 2, pp. 189-201, 2009.

[21] J. R. A. Schafer, B. M. Jesdale, J. A. George, N. M. Kouttab, and A. S. De Groot, "Prediction of well-conserved HIV-1 ligands using a matrix-based algorithm, EpiMatrix," Vaccine, vol. 16, no. 19, pp. 1880-1884, 1998.

[22] C. A. Weber, P. J. Mehta, M. Ardito, L. Moise, B. Martin, and A. S. De Groot, "T cell epitope: friend or foe? Immunogenicity of biologics in context," Advanced Drug Delivery Reviews, vol. 61, no. 11, pp. 965-976, 2009.

[23] D. T. Golan and Y. Borel, "Nonantigenicity and immunologic tolerance: the role of the carrier in the induction of tolerance to the hapten," Journal of Experimental Medicine, vol. 134, no. 4, pp. 1046-1061, 1971.

[24] E. T. Zambidis and D. W. Scott, "Epitope-specific tolerance induction with an engineered immunoglobulin," Proceedings of the National Academy of Sciences of the United States of America, vol. 93, no. 10, pp. 5019-5024, 1996.

[25] N. Soukhareva, Y. Jiang, and D. W. Scott, “Treatment of diabetes in NOD mice by gene transfer of Ig-fusion proteins into B cells: role of T regulatory cells," Cellular Immunology, vol. 240, no. 1, pp. 41-46, 2006.

[26] L. Song, J. Wang, R. Wang et al., "Retroviral delivery of GADIgG fusion construct induces tolerance and modulates diabetes: a role for $\mathrm{CD}^{+}$regulatory T cells and TGF- $\beta$ ?" Gene Therapy, vol. 11, no. 20, pp. 1487-1496, 2004. 
[27] A. Lobell, R. Weissert, M. K. Storch et al., "Vaccination with DNA encoding an immunodominant myelin basic protein peptide targeted to Fc of immunoglobulin G suppresses experimental autoimmune encephalomyelitis," Journal of Experimental Medicine, vol. 187, no. 9, pp. 1543-1548, 1998.

[28] Y. Su, G. Carey, M. Marić, and D. W. Scott, "B cells induce tolerance by presenting endogenous peptide-IgG on MHC class II molecules via an IFN- $\gamma$-inducible lysosomal thiol reductasedependent pathway," Journal of Immunology, vol. 181, no. 2, pp. 1153-1160, 2008.

[29] M. El-Amine, J. A. Hinshaw, and D. W. Scott, "In vivo induction of tolerance by an lg peptide is not affected by the deletion of FcR or a mutated lgG Fc fragment," International Immunology, vol. 14, no. 7, pp. 761-766, 2002.

[30] J. Bayry, K. Bansal, M. D. Kazatchkine, and S. V. Kaveri, "DCSIGN and $\alpha 2,6$-sialylated IgG Fc interaction is dispensable for the anti-inflammatory activity of IVIg on human dendritic cells," Proceedings of the National Academy of Sciences of the United States of America, vol. 106, no. 9, p. E24, 2009.

[31] R. M. Anthony, F. Nimmerjahn, D. J. Ashline, V. N. Reinhold, J. C. Paulson, and J. V. Ravetch, "Recapitulation of IVIG antiinflammatory activity with a recombinant IgG Fc," Science, vol. 320, no. 5874, pp. 373-376, 2008.

[32] R. Bazin, R. Lemieux, and T. Tremblay, "Reversal of immune thrombocytopenia in mice by cross-linking human immunoglobulin $\mathrm{G}$ with a high-affinity monoclonal antibody," British Journal of Haematology, vol. 135, no. 1, pp. 97-100, 2006.

[33] X. Yu, S. Vasiljevic, D. A. Mitchell, M. Crispin, and C. N. Scanlan, "Dissecting the molecular mechanism of IVIg therapy: the interaction between serum IgG and DC-SIGN is independent of antibody glycoform or Fc domain," Journal of Molecular Biology, vol. 425, no. 8, pp. 1253-1258, 2013.

[34] D. A. Patel, A. Puig-Canto, D. K. Challa, H. P. Montoyo, R. J. Ober, and E. S. Ward, "Neonatal Fc receptor blockade by Fc engineering ameliorates arthritis in a murine model," Journal of Immunology, vol. 187, no. 2, pp. 1015-1022, 2011.

[35] J. G. Routsias, N. C. Kyriakidis, D. M. Friedman et al., "Association of the idiotype:Antiidiotype antibody ratio with the efficacy of intravenous immunoglobulin treatment for the prevention of recurrent autoimmune-associated congenital heart block," Arthritis and Rheumatism, vol. 63, no. 9, pp. 2783-2789, 2011.

[36] S. C. Jordan, M. Toyoda, and A. A. Vo, "Regulation of immunity and inflammation by intravenous immunoglobulin: relevance to solid organ transplantation," Expert Review of Clinical Immunology, vol. 7, no. 3, pp. 341-348, 2011.

[37] A. Durandy, S. V. Kaveri, T. W. Kuijpers et al., "Intravenous immunoglobulins-understanding properties and mechanisms," Clinical and Experimental Immunology, vol. 158, no. 1, pp. 2-13, 2009.

[38] M. S. Maddur, P. Hegde, M. Sharma, S. V. Kaveri, and J. Bayry, "B cells are resistant to immunomodulation by "IVIg-educated" dendritic cells," Autoimmunity Reviews, vol. 11, no. 2, pp. 154156, 2011.

[39] M. S. Maddur, S. V. Kaveri, and J. Bayry, "Comparison of different IVIg preparations on IL-17 production by human Th17 cells," Autoimmunity Reviews, vol. 10, no. 12, pp. 809-810, 2011.

[40] A. Kessel, H. Ammuri, R. Peri et al., "Intravenous immunoglobulin therapy affects $\mathrm{T}$ regulatory cells by increasing their suppressive function," Journal of Immunology, vol. 179, no. 8, pp. 5571-5575, 2007.
[41] A. H. Massoud, M. Yona, D. Xue et al., "Dendritic cell immunoreceptor: a novel receptor for intravenous immunoglobulin mediates induction of regulatory T cells," The Journal of Allergy and Clinical Immunolog, 2013.

[42] M. Lopez, M. R. Clarkson, M. Albin, M. H. Sayegh, and N. Najafian, "A novel mechanism of action for anti-thymocyte globulin: induction of $\mathrm{CD} 4^{+} \mathrm{CD} 25^{+} \mathrm{Foxp}^{+}$regulatory T cells," Journal of the American Society of Nephrology, vol. 17, no. 10, pp. 2844-2853, 2006.

[43] T. Tha-In, H. J. Metselaar, A. R. Bushell, J. Kwekkeboom, and K. J. Wood, "Intravenous immunoglobulins promote skin allograft acceptance by triggering functional activation of CD4+Foxp3+ T cells," Transplantation, vol. 89, no. 12, pp. 1446-1455, 2010.

[44] K. L. Legge, J. J. Bell, L. Li, R. Gregg, J. C. Caprio, and H. Zaghouani, "Multi-modal antigen specific therapy for autoimmunity," International Reviews of Immunology, vol. 20, no. 5, pp. 593-611, 2001.

[45] H. Orbach, U. Katz, Y. Sherer, and Y. Shoenfeld, "Intravenous immunoglobulin: adverse effects and safe administration," Clinical Reviews in Allergy and Immunology, vol. 29, no. 3, pp. 173184, 2005.

[46] B. D. Mazer, S. Al-Tamemi, J. W. Yu, and Q. Hamid, "Immune supplementation and immune modulation with intravenous immunoglobulin," Journal of Allergy and Clinical Immunology, vol. 116, no. 4, pp. 941-944, 2005.

[47] J. S. Orange, E. M. Hossny, C. R. Weiler et al., "Use of intravenous immunoglobulin in human disease: a review of evidence by members of the Primary Immunodeficiency Committee of the American Academy of Allergy, Asthma and Immunology," Journal of Allergy and Clinical Immunology, vol. 117, no. 4, pp. S525-S553, 2006. 


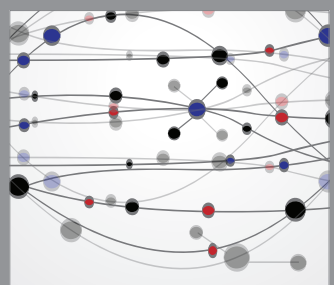

The Scientific World Journal
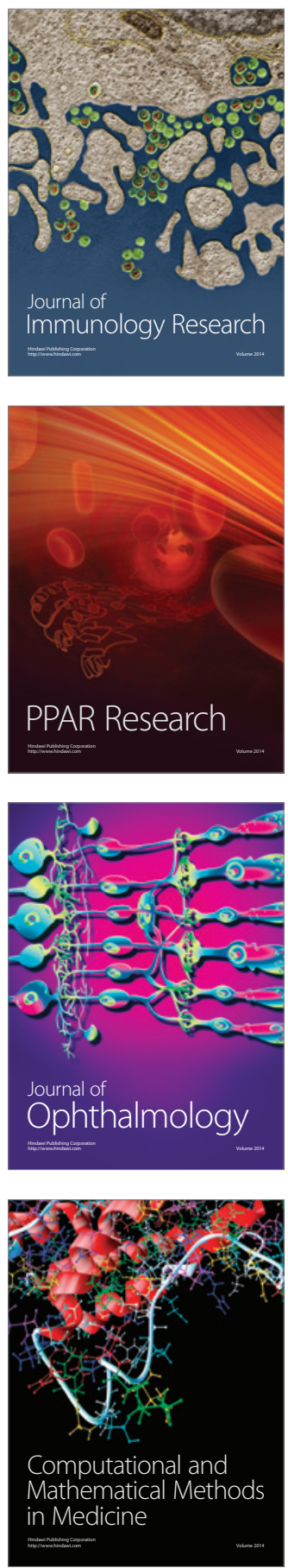

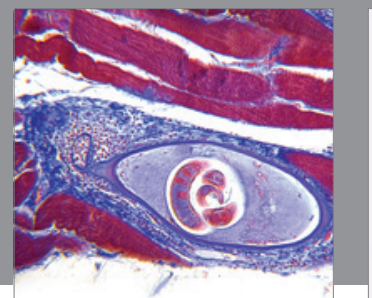

Gastroenterology

Research and Practice
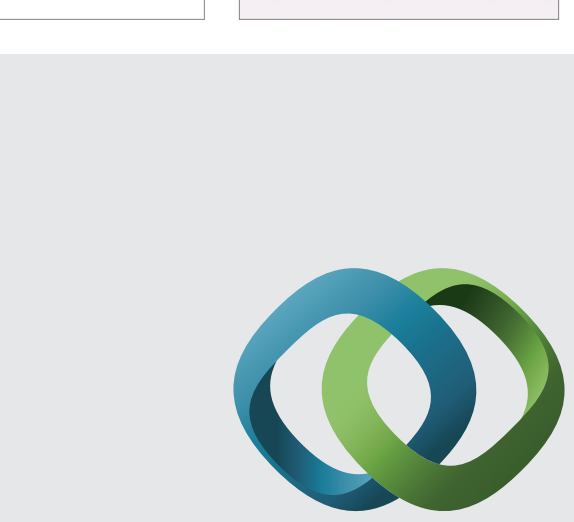

\section{Hindawi}

Submit your manuscripts at

http://www.hindawi.com
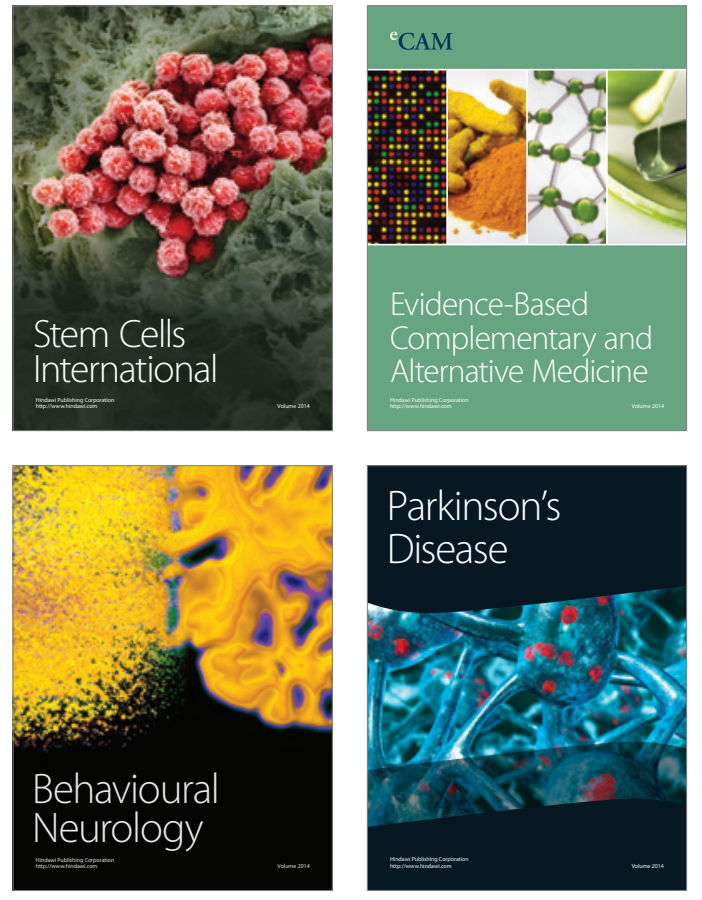
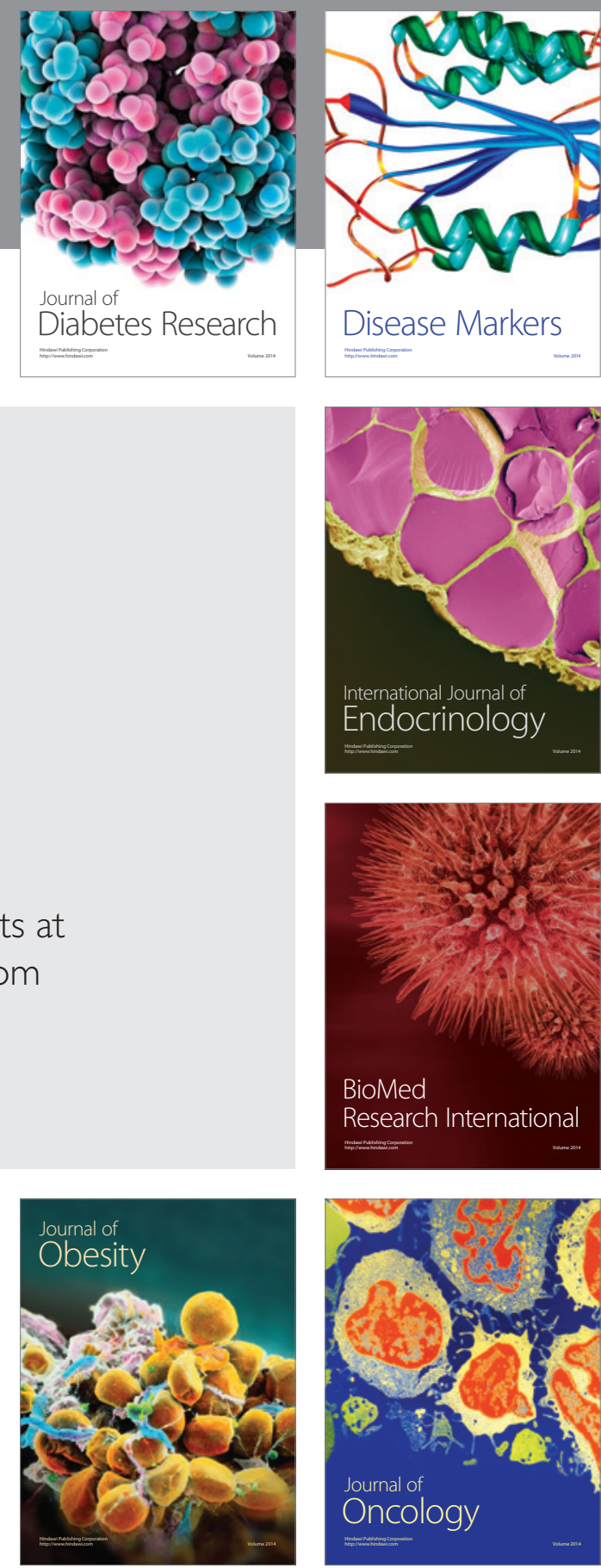

Disease Markers
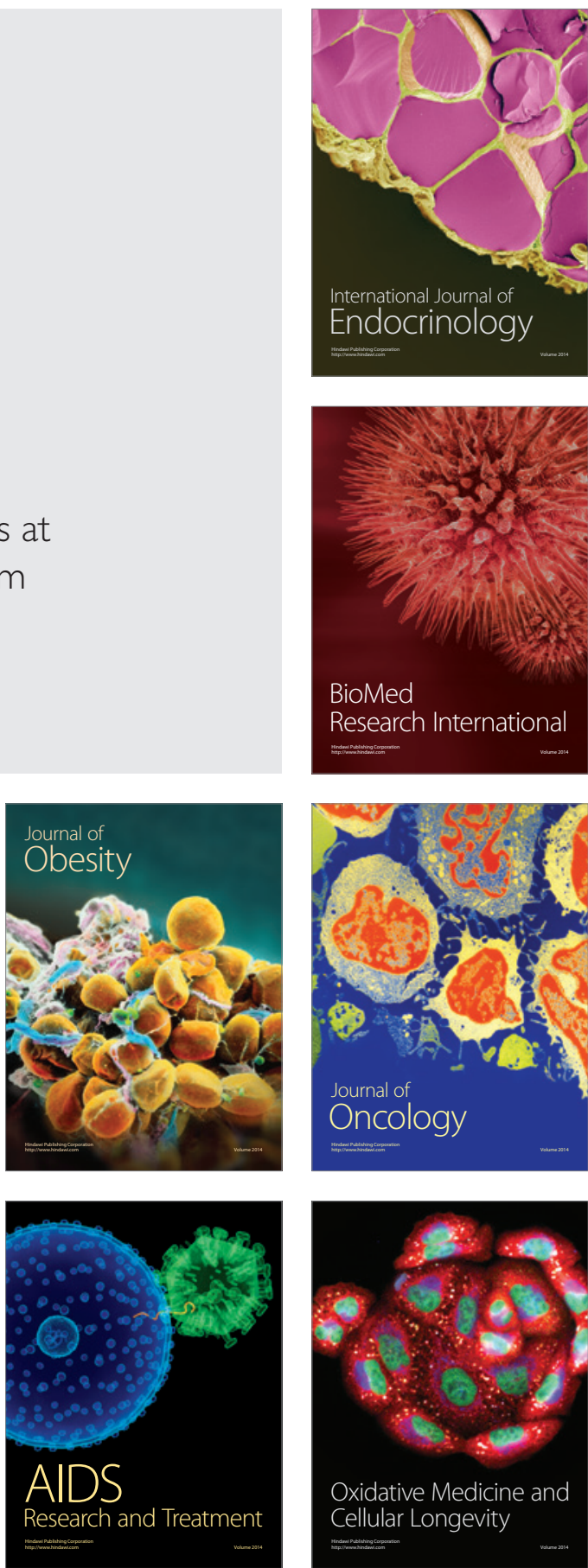